\title{
SUPPLEMENTARY MATERIAL FOR: ESTIMATION OF (NEAR) LOW-RANK MATRICES WITH NOISE AND HIGH-DIMENSIONAL SCALING
}

\author{
By Sahand Negahban and Martin J. Wainwright \\ University of California, Berkeley
}

\section{APPENDIX A: INTRODUCTION}

In this supplement we present many of the technical details from the main work [1]. Equation or theorem references made to the main document are relative to the numbering scheme of the document and will not contain letters.

\section{APPENDIX B: PROOF OF LEMMA 1}

Part (a) of the claim was proved in Recht et al. [2]; we simply provide a proof here for completeness. We write the SVD as $\Theta^{*}=U D V^{T}$, where $U \in \mathbb{R}^{m_{1} \times m_{1}}$ and $V \in \mathbb{R}^{m_{2} \times m_{2}}$ are orthogonal matrices, and $D$ is the matrix formed by the singular values of $\Theta^{*}$. Note that the matrices $U^{r}$ and $V^{r}$ are given by the first $r$ columns of $U$ and $V$ respectively. We then define the matrix $\Gamma=U^{T} \Delta V \in \mathbb{R}^{m_{1} \times m_{2}}$, and write it in block form as

$$
\Gamma=\left[\begin{array}{ll}
\Gamma_{11} & \Gamma_{12} \\
\Gamma_{21} & \Gamma_{22}
\end{array}\right], \quad \text { where } \Gamma_{11} \in \mathbb{R}^{r \times r} \text {, and } \Gamma_{22} \in \mathbb{R}^{\left(m_{1}-r\right) \times\left(m_{2}-r\right)} .
$$

We now define the matrices

$$
\Delta^{\prime \prime}=U\left[\begin{array}{cc}
0 & 0 \\
0 & \Gamma_{22}
\end{array}\right] V^{T}, \quad \text { and } \Delta^{\prime}=\Delta-\Delta^{\prime \prime} .
$$

Note that we have

$$
\operatorname{rank}\left(\Delta^{\prime}\right)=\operatorname{rank}\left[\begin{array}{cc}
\Gamma_{11} & \Gamma_{12} \\
\Gamma_{21} & 0
\end{array}\right] \leq \operatorname{rank}\left[\begin{array}{cc}
\Gamma_{11} & \Gamma_{12} \\
0 & 0
\end{array}\right]+\operatorname{rank}\left[\begin{array}{cc}
\Gamma_{11} & 0 \\
\Gamma_{21} & 0
\end{array}\right] \leq 2 r
$$

which establishes Lemma 1(a). Moreover, we note for future reference that by construction of $\Delta^{\prime \prime}$, the nuclear norm satisfies the decomposition

$$
\left\|\Pi_{\mathcal{A}^{r}}\left(\Theta^{*}\right)+\Delta^{\prime \prime}\right\|_{1}=\left\|\Pi_{\mathcal{A}^{r}}\left(\Theta^{*}\right)\right\|_{1}+\left\|\Delta^{\prime \prime}\right\|_{1} .
$$


We now turn to the proof of Lemma 1(b). Recall that the error $\Delta=\widehat{\Theta}-\Theta^{*}$ associated with any optimal solution must satisfy the inequality (30), which implies that

$0 \leq \frac{1}{N}\langle\vec{\varepsilon}, \mathfrak{X}(\Delta)\rangle+\lambda_{N}\left\{\left\|\Theta^{*}\right\|_{1}-\|\widehat{\Theta}\|_{1}\right\} \leq\left\|\frac{1}{N} \mathfrak{X}^{*}(\vec{\varepsilon})\right\|_{\text {op }}\|\Delta\|_{1}+\lambda_{N}\left\{\left\|\Theta^{*}\right\|_{1}-\|\widehat{\Theta}\|_{1}\right\}$, where we have used the bound (31).

Note that we have the decomposition $\Theta^{*}=\Pi_{\mathcal{A}^{r}}\left(\Theta^{*}\right)+\Pi_{\mathcal{B}^{r}}\left(\Theta^{*}\right)$. Using this decomposition, the triangle inequality and the relation (B.1), we have

$$
\begin{aligned}
\|\widehat{\Theta}\|_{1} & =\left\|\left(\Pi_{\mathcal{A}^{r}}\left(\Theta^{*}\right)+\Delta^{\prime \prime}\right)+\left(\Pi_{\mathcal{B}^{r}}\left(\Theta^{*}\right)+\Delta^{\prime}\right)\right\|_{1} \\
& \geq\left\|\left(\Pi_{\mathcal{A}^{r}}\left(\Theta^{*}\right)+\Delta^{\prime \prime}\right)\right\|_{1}-\left\|\left(\Pi_{\mathcal{B}^{r}}\left(\Theta^{*}\right)+\Delta^{\prime}\right)\right\|_{1} \\
& \geq\left\|\Pi_{\mathcal{A}^{r}}\left(\Theta^{*}\right)\right\|_{1}+\left\|\Delta^{\prime \prime}\right\|_{1}-\left\{\|\left(\Pi_{\mathcal{B}^{r}}\left(\Theta^{*}\right)\left\|_{1}+\right\| \Delta^{\prime} \|_{1}\right\} .\right.
\end{aligned}
$$

Consequently, we have

$$
\begin{aligned}
\left\|\Theta^{*}\right\|_{1}-\|\widehat{\Theta}\|_{1} & \leq\left\|\Theta^{*}\right\|_{1}-\left\{\left\|\Pi_{\mathcal{A}^{r}}\left(\Theta^{*}\right)\right\|_{1}+\left\|\Delta^{\prime \prime}\right\|_{1}\right\}+\left\{\|\left(\Pi_{\mathcal{B}^{r}}\left(\Theta^{*}\right)\left\|_{1}+\right\| \Delta^{\prime} \|_{1}\right\}\right. \\
& =2\left\|\Pi_{\mathcal{B}^{r}}\left(\Theta^{*}\right)\right\|_{1}+\left\|\Delta^{\prime}\right\|_{1}-\left\|\Delta^{\prime \prime}\right\|_{1} .
\end{aligned}
$$

Substituting this inequality into the bound (B.2), we obtain

$$
0 \leq\left\|\frac{1}{N} \mathfrak{X}^{*}(\vec{\varepsilon})\right\|_{\text {op }}\|\Delta\|_{1}+\lambda_{N}\left\{2\left\|\Pi_{\mathcal{B}^{r}}\left(\Theta^{*}\right)\right\|_{1}+\left\|\Delta^{\prime}\right\|_{1}-\left\|\Delta^{\prime \prime}\right\|_{1}\right\} .
$$

Finally, since $\left\|\frac{1}{N} \mathfrak{X}^{*}(\vec{\varepsilon})\right\|_{\text {op }} \leq \lambda_{N} / 2$ by assumption, we conclude that

$$
0 \leq \lambda_{N}\left\{2\left\|\Pi_{\mathcal{B}^{r}}\left(\Theta^{*}\right)\right\|_{1}+\frac{3}{2}\left\|\Delta^{\prime}\right\|_{1}-\frac{1}{2}\left\|\Delta^{\prime \prime}\right\|_{1}\right\} .
$$

Since $\left\|\Pi_{\mathcal{B}^{r}}\left(\Theta^{*}\right)\right\|_{1}=\sum_{j=r+1}^{m} \sigma_{j}\left(\Theta^{*}\right)$, the bound (32) follows.

\section{APPENDIX C: PROOF OF COROLLARY 5}

Recall that for this model, the observations are of the form $y_{i}=\left\langle\left\langle X_{i}, \Theta^{*}\right\rangle\right\rangle+$ $\varepsilon_{i}$, where $\Theta^{*} \in \mathbb{R}^{m_{1} \times m_{2}}$ is the unknown matrix, and $\left\{\varepsilon_{i}\right\}_{i=1}^{N}$ is an associated noise sequence.

We now show how Proposition 1 implies the RSC property with an appropriate tolerance parameter $\delta>0$ to be defined. Observe that the bound (25) implies that for any $\Delta \in \mathcal{C}$, we have

$$
\begin{aligned}
\frac{\|\mathfrak{X}(\Delta)\|_{2}}{\sqrt{N}} & \geq \frac{\sqrt{\sigma_{\min }(\Sigma)}}{4}\|\Delta\|_{F}-12 \rho(\Sigma)\left(\sqrt{\frac{m_{1}}{N}}+\sqrt{\frac{m_{2}}{N}}\right)\|\Delta\|_{1} \\
& =\frac{\sqrt{\sigma_{\min }(\Sigma)}}{4}\{\|\Delta\|_{F}-\underbrace{\frac{48 \rho(\Sigma)}{\sqrt{\sigma_{\min }(\Sigma)}}\left(\sqrt{\frac{m_{1}}{N}}+\sqrt{\frac{m_{2}}{N}}\right)}_{\tau}\|\Delta\|_{1}\},
\end{aligned}
$$


where we have defined the quantity $\tau>0$. Following the arguments used in the proofs of Theorem 1 and Corollary 2, we find that

$$
\|\Delta\|_{1} \leq 4\left\|\Delta^{\prime}\right\|_{1}+4 \sum_{j=r+1}^{m} \sigma_{j}\left(\Theta^{*}\right) \leq 4 \sqrt{2 R_{q} \tau^{-q}}\left\|\Delta^{\prime}\right\|_{F}+4 R_{q} \tau^{1-q} .
$$

Note that this corresponds to truncating the matrices at effective rank $r=$ $2 R_{q} \tau^{-q}$. Combining this bound with the definition of $\tau$, we obtain

$\tau\|\Delta\|_{1} \leq 4 \sqrt{2 R_{q}} \tau^{1-q / 2}\left\|\Delta^{\prime}\right\|_{F}+4 R_{q} \tau^{2-q} \leq 4 \sqrt{2 R_{q}} \tau^{1-q / 2}\|\Delta\|_{F}+4 R_{q} \tau^{2-q}$.

Substituting this bound into equation (C.1) yields

$$
\frac{\|\mathfrak{X}(\Delta)\|_{2}}{\sqrt{N}} \geq \frac{\sqrt{\sigma_{\min }(\Sigma)}}{4}\left\{\|\Delta\|_{F}-4 \sqrt{2 R_{q}} \tau^{1-q / 2}\left\|\Delta^{\prime}\right\|_{F}-4 R_{q} \tau^{2-q}\right\} .
$$

As long $N>c_{0} R_{q}^{2 /(2-q)} \frac{\rho^{2}(\Sigma)}{\sigma_{\min }(\Sigma)}\left(m_{1}+m_{2}\right)$ for a sufficiently large constant $c_{0}$, we can ensure that $4 \sqrt{2 R_{q}} \tau^{1-q / 2}<1 / 2$, and hence that

$$
\frac{\|\mathfrak{X}(\Delta)\|_{2}}{\sqrt{N}} \geq \frac{\sqrt{\sigma_{\min }(\Sigma)}}{4}\left\{\frac{1}{2}\|\Delta\|_{F}-4 R_{q} \tau^{2-q}\right\} .
$$

Consequently, if we define $\delta:=16 R_{q} \tau^{2-q}$, then we are guaranteed that for all $\|\Delta\|_{F} \geq \delta$, we have $4 R_{q} \tau^{2-q} \leq\|\Delta\|_{F} / 4$, and hence

$$
\frac{\|\mathfrak{X}(\Delta)\|_{2}}{\sqrt{N}} \geq \frac{\sqrt{\sigma_{\min }(\Sigma)}}{16}\|\Delta\|_{F}
$$

for all $\|\Delta\|_{F} \geq \delta$. We have thus shown that $\mathcal{C}\left(2 R_{q} \tau^{-q} ; \delta\right)$ with parameter $\kappa(\mathfrak{X})=\frac{\sigma_{\min }(\Sigma)}{256}$.

The next step is to control the quantity $\left\|\mathfrak{X}^{*}(\vec{\varepsilon})\right\|_{\text {op }} / N$, required for specifying a suitable choice of $\lambda_{N}$.

Lemma C.1. If $\|\vec{\varepsilon}\|_{2} \leq 2 \nu \sqrt{N}$, then there are universal constants $c_{i}$ such that

(C.3) $\mathbb{P}\left[\frac{\left\|\mathfrak{X}^{*}(\vec{\varepsilon})\right\|_{\mathrm{op}}}{N} \geq c_{0} \rho(\Sigma) \nu\left(\sqrt{\frac{m_{1}}{N}}+\sqrt{\frac{m_{2}}{N}}\right)\right] \leq c_{1} \exp \left(-c_{2}\left(m_{1}+m_{2}\right)\right)$. 
Proof. By the definition of the adjoint operator, we have $Z=\frac{1}{N} \mathfrak{X}^{*}(\vec{\varepsilon})=$ $\frac{1}{N} \sum_{i=1}^{N} \varepsilon_{i} X_{i}$. Since the observation matrices $\left\{X_{i}\right\}_{i=1}^{N}$ are i.i.d. Gaussian, if the sequence $\left\{\varepsilon_{i}\right\}_{i=1}^{N}$ is viewed as fixed (by conditioning as needed), then the random matrix $Z$ is a sample from the $\Gamma$-ensemble with covariance matrix $\Gamma=\frac{\|\vec{\varepsilon}\|^{2}}{N^{2}} \Sigma \preceq \frac{2 \nu^{2}}{N} \Sigma$. Therefore, letting $\widetilde{Z} \in \mathbb{R}^{m_{1} \times m_{2}}$ be a random matrix drawn from the $2 \nu^{2} \Sigma / N$-ensemble, we have

$$
\mathbb{P}\left[\|Z\|_{\text {op }} \geq t\right] \leq \mathbb{P}\left[\|\widetilde{Z}\|_{\text {op }} \geq t\right] .
$$

Using Lemma H.1 from Appendix H, we have

$$
\mathbb{E}\left[\|\widetilde{Z}\|_{\text {op }}\right] \leq \frac{12 \sqrt{2} \nu \rho(\Sigma)}{\sqrt{N}}\left(\sqrt{m_{1}}+\sqrt{m_{2}}\right)
$$

and

$$
\mathbb{P}\left[\|\widetilde{Z}\|_{\text {op }} \geq \mathbb{E}\left[\|\widetilde{Z}\|_{\text {op }}\right]+t\right] \leq \exp \left(-c_{1} \frac{N t^{2}}{\nu^{2} \rho^{2}(\Sigma)}\right)
$$

for a universal constant $c_{1}$. Setting $t^{2}=\Omega\left(\frac{\nu^{2} \rho^{2}(\Sigma)\left(\sqrt{m_{1}}+\sqrt{m_{2}}\right)^{2}}{N}\right.$ yields the claim.

\section{APPENDIX D: PROOF OF COROLLARY 6}

This corollary follows from a combination of Proposition 1 and Lemma 1. Let $\widehat{\Theta}$ be an optimal solution to the $\operatorname{SDP}(29)$, and let $\Delta=\widehat{\Theta}-\Theta^{*}$ be the error. Since $\widehat{\Theta}$ is optimal and $\Theta^{*}$ is feasible for the SDP, we have $\|\widehat{\Theta}\|_{1}=$ $\left\|\Theta^{*}+\Delta\right\|_{1} \leq\left\|\Theta^{*}\right\|_{1}$. Using the decomposition $\Delta=\Delta^{\prime}+\Delta^{\prime \prime}$ from Lemma 1 and applying triangle inequality, we have

$$
\left\|\Theta^{*}+\Delta^{\prime}+\Delta^{\prime \prime}\right\|_{1} \geq\left\|\Theta^{*}+\Delta^{\prime \prime}\right\|_{1}-\left\|\Delta^{\prime}\right\|_{1} .
$$

From the properties of the decomposition in Lemma 1 (see Appendix B), we find that

$$
\|\widehat{\Theta}\|_{1}=\left\|\Theta^{*}+\Delta^{\prime}+\Delta^{\prime \prime}\right\|_{1} \geq\left\|\Theta^{*}\right\|_{1}+\left\|\Delta^{\prime \prime}\right\|_{1}-\left\|\Delta^{\prime}\right\|_{1} .
$$

Combining the pieces yields that $\left\|\Delta^{\prime \prime}\right\|_{1} \leq\left\|\Delta^{\prime}\right\|_{1}$, and hence $\|\Delta\|_{1} \leq 2\left\|\Delta^{\prime}\right\|_{1}$. By Lemma 1(a), the rank of $\Delta^{\prime}$ is at most $2 r$, so that we obtain $\|\Delta\|_{1} \leq$ $2 \sqrt{2 r}\|\Delta\|_{F} \leq 4 \sqrt{r}\|\Delta\|_{F}$. 
Note that $\mathfrak{X}(\Delta)=0$, since both $\widehat{\Theta}$ and $\Theta^{*}$ agree with the observations. Consequently, from Proposition 1, we have that

$$
\begin{aligned}
0=\frac{\|\mathfrak{X}(\Delta)\|_{2}}{\sqrt{N}} & \geq \frac{1}{4}\|\Delta\|_{F}-12 \rho(\Sigma)\left(\sqrt{\frac{m_{1}}{N}}+\sqrt{\frac{m_{2}}{N}}\right)\|\Delta\|_{1} \\
& \geq\|\Delta\|_{F}\left(\frac{1}{4}-12 \rho(\Sigma) \sqrt{\frac{r m_{1}}{N}}+12 \rho(\Sigma) \sqrt{\frac{r m_{2}}{N}}\right) \\
& \geq \frac{1}{20}\|\Delta\|_{F}
\end{aligned}
$$

where the final inequality as long as $N>c_{0} \rho^{2}(\Sigma) r\left(m_{1}+m_{2}\right)$ for a sufficiently large constant $c_{0}$. We have thus shown that $\Delta=0$, which implies that $\widehat{\Theta}=\Theta^{*}$ as claimed.

\section{APPENDIX E: CONSISTENCY IN OPERATOR NORM}

In this appendix, we derive a bound on the operator norm error for both the low-rank multivariate regression and auto-regressive model estimation problems. In this statement, it is convenient to specify these models in the form $Y=X \Theta^{*}+W$, where $Y \in \mathbb{R}^{n \times m_{2}}$ is a matrix of observations.

Proposition E.1 (Operator norm consistency). Consider the multivariate regression problem and the SDP under the conditions of Corollary 3. Then any solution $\widehat{\Theta}$ to the SDP satisfies the bound

$$
\left\|\widehat{\Theta}-\Theta^{*}\right\|_{\text {op }} \leq c^{\prime} \frac{\nu \sqrt{\sigma_{\max }(\Sigma)}}{\sigma_{\min }(\Sigma)} \sqrt{\frac{m_{1}+m_{2}}{n}} .
$$

We note that a similar bound applies to the auto-regressive model treated in Corollary 4.

Proof. For any subgradient matrix $Z \in \partial\|\widehat{\Theta}\|_{1}$, we are guaranteed $\|Z\|_{\text {op }} \leq 1$. Furthermore, by the KKT conditions [3] for the nuclear norm SDP, any solution $\widehat{\Theta}$ must satisfy the condition

$$
\frac{1}{n} X^{T} X \widehat{\Theta}-\frac{X^{T} Y}{n}+\lambda_{n} Z=0 .
$$

Hence, simple algebra and the triangle inequality yield that

$$
\|\widehat{\Theta}\|_{\text {op }} \leq\left\|\left(\frac{1}{n} X^{T} X\right)^{-1}\right\|_{\mathrm{op}}\left[\left\|X^{T} W / n\right\|_{\mathrm{op}}+\lambda_{n}\right] .
$$

imsart-aos ver. 2010/08/03 file: RR_AOS_Sahand_trim.tex date: November 16, 2010 
Lemma 2 yields that $\left\|\left(\frac{1}{n} X^{T} X\right)^{-1}\right\|_{\text {op }} \leq \frac{9}{\sigma_{\min }(\Sigma)}$ with high probability. Combining these inequalities yields

$$
\|\widehat{\Theta}\|_{\text {op }} \leq c_{1} \frac{\lambda_{n}}{\sigma_{\min }(\Sigma)} .
$$

We require that $\lambda_{n} \geq 2\left\|X^{T} W\right\|_{\text {op }} / n$. From Lemma 3 , it suffices to set $\lambda_{n} \geq$ $c_{0} \sqrt{\sigma_{\max }(\Sigma)} \nu \sqrt{\frac{m_{1}+m_{2}}{n}}$. Combining the pieces yields the claim.

\section{APPENDIX F: PROOF OF LEMMA 3}

Let $S^{m-1}=\left\{u \in \mathbb{R}^{m} \mid\|u\|_{2}=1\right\}$ denote the Euclidean sphere in $m$ dimensions. The operator norm of interest has the variational representation

$$
\frac{1}{n}\left\|X^{T} W\right\|_{\mathrm{op}}=\frac{1}{n} \sup _{u \in S^{m_{1}-1}} \sup _{v \in S^{m_{2}-1}} v^{T} X^{T} W u
$$

For positive scalars $a$ and $b$, define the (random) quantity

$$
\Psi(a, b):=\sup _{u \in a S^{m_{1}-1}} \sup _{v \in b S^{m_{2}-1}}\langle X v, W u\rangle .
$$

and note that our goal is to upper bound $\Psi(1,1)$. Note moreover that $\Psi(a, b)=a b \Psi(1,1)$, a relation which will be useful in the analysis.

Let $\mathcal{A}=\left\{u^{1}, \ldots, u^{A}\right\}$ and $\mathcal{B}=\left\{v^{1}, \ldots, v^{B}\right\}$ denote $1 / 4$ coverings of $S^{m_{1}-1}$ and $S^{m_{2}-1}$, respectively. We now claim that we have the upper bound

$$
\Psi(1,1) \leq 4 \max _{u^{a} \in \mathcal{A}, v^{b} \in \mathcal{B}}\left\langle X v^{b}, W u^{a}\right\rangle
$$

To establish this claim, we note that since the sets $\mathcal{A}$ and $\mathcal{B}$ are $1 / 4$-covers, for any pair $(u, v) \in S^{m-1} \times S^{m-1}$, there exists a pair $\left(u^{a}, v^{b}\right) \in \mathcal{A} \times \mathcal{B}$ such that $u=u^{a}+\Delta u$ and $v=v^{b}+\Delta v$, with $\max \left\{\|\Delta u\|_{2},\|\Delta v\|_{2}\right\} \leq 1 / 4$. Consequently, we can write

$$
\langle X v, W u\rangle=\left\langle X v^{b}, W u^{a}\right\rangle+\left\langle X v^{b}, W \Delta u\right\rangle+\left\langle X \Delta v, W u^{a}\right\rangle+\langle X \Delta v, W \Delta u\rangle .
$$

By construction, we have the bound $\left|\left\langle X v^{b}, W \Delta u\right\rangle\right| \leq \Psi(1,1 / 4)=\frac{1}{4} \Psi(1,1)$, and similarly $\left|\left\langle X \Delta v, W u^{a}\right\rangle\right| \leq \frac{1}{4} \Psi(1,1)$ as well as $|\langle X \Delta v, W \Delta u\rangle| \leq \frac{1}{16} \Psi(1,1)$. Substituting these bounds into the decomposition (F.2) and taking suprema over the left and right-hand sides, we conclude that

$$
\Psi(1,1) \leq \max _{u^{a} \in \mathcal{A}, v^{b} \in \mathcal{B}}\left\langle X v^{b}, W u^{a}\right\rangle+\frac{9}{16} \Psi(1,1)
$$


from which the bound (F.1) follows.

We now apply the union bound to control the discrete maximum. It is known (e.g., $[4,5])$ that there exists a $1 / 4$ covering of $S^{m_{1}-1}$ and $S^{m_{2}-1}$ with at most $A \leq 8^{m_{1}}$ and $B \leq 8^{m_{2}}$ elements respectively. Consequently, we have

$$
\mathbb{P}[|\Psi(1,1)| \geq 4 \delta n] \leq 8^{m_{1}+m_{2}} \max _{u^{a}, v^{b}} \mathbb{P}\left[\frac{\left|\left\langle X v^{b}, W u^{a}\right\rangle\right|}{n} \geq \delta\right] .
$$

It remains to obtain a good bound on the quantity $\frac{1}{n}\langle X v, W u\rangle=\frac{1}{n} \sum_{i=1}^{n}\left\langle v, X_{i}\right\rangle\left\langle u, W_{i}\right\rangle$, where $(u, v) \in S^{m_{1}-1} \times S^{m_{2}-1}$ are arbitrary but fixed. Since $W_{i} \in \mathbb{R}^{m_{1}}$ has i.i.d. $N\left(0, \nu^{2}\right)$ elements and $u$ is fixed, we have $Z_{i}:=\left\langle u, W_{i}\right\rangle \sim N\left(0, \nu^{2}\right)$ for each $i=1, \ldots, n$. These variables are independent of one another, and of the random matrix $X$. Therefore, conditioned on $X$, the sum $Z:=$ $\frac{1}{n} \sum_{i=1}^{n}\left\langle v, X_{i}\right\rangle\left\langle u, W_{i}\right\rangle$ is zero-mean Gaussian with variance

$$
\alpha^{2}:=\frac{\nu^{2}}{n}\left(\frac{1}{n}\|X v\|_{2}^{2}\right) \leq \frac{\nu^{2}}{n}\left\|X^{T} X / n\right\|_{\mathrm{op}} .
$$

Define the event $\mathcal{T}=\left\{\alpha^{2} \leq \frac{9 \nu^{2}\|\Sigma\|_{\text {op }}}{n}\right\}$. Using Lemma 2 , we have $\left\|X^{T} X / n\right\|_{\text {op }} \leq$ $9 \sigma_{\max }(\Sigma)$ with probability at least $1-2 \exp (-n / 2)$, which implies that $\mathbb{P}\left[\mathcal{T}^{c}\right] \leq 2 \exp (-n / 2)$. Therefore, conditioning on the event $\mathcal{T}$ and its complement $\mathcal{T}^{c}$, we obtain

$$
\begin{aligned}
\mathbb{P}[|Z| \geq t] & \leq \mathbb{P}[|Z| \geq t \mid \mathcal{T}]+\mathbb{P}\left[\mathcal{T}^{c}\right] \\
& \leq \exp \left(-n \frac{t^{2}}{2 \nu^{2}\left(4+\|\Sigma\|_{\text {op }}\right)}\right)+2 \exp (-n / 2) .
\end{aligned}
$$

Combining this tail bound with the upper bound (F.3), we have

$$
\mathbb{P}[|\psi(1,1)| \geq 4 \delta n] \leq 8^{m_{1}+m_{2}}\left\{\exp \left(-n \frac{t^{2}}{18 \nu^{2}\|\Sigma\|_{\text {op }}}\right)+2 \exp (-n / 2)\right\} .
$$

Setting $t^{2}=20 \nu^{2}\|\Sigma\|_{\text {op }} \frac{m_{1}+m_{2}}{n}$, this probability vanishes as long as $n>$ $16\left(m_{1}+m_{2}\right)$.

\section{APPENDIX G: TECHNICAL DETAILS FOR COROLLARY 4}

In this appendix, we collect the proofs of Lemmas 4 and 5 . 
G.1. Proof of Lemma 4. Recalling that $S^{m-1}$ denotes the unit-norm Euclidean sphere in $m$-dimensions, we first observe that $\|X\|_{\text {op }}=\sup _{u \in S^{m-1}}\|X u\|_{2}$. Our next step is to reduce the supremum to a maximization over a finite set, using a standard covering argument. Let $\mathcal{A}=\left\{u^{1}, \ldots, u^{A}\right\}$ denote a $1 / 2$ cover of it. By definition, for any $u \in S^{m-1}$, there is some $u^{a} \in \mathcal{A}$ such that $u=u^{a}+\Delta u$, where $\|\Delta u\|_{2} \leq 1 / 2$. Consequently, for any $u \in S^{m-1}$, the triangle inequality implies that

$$
\|X u\|_{2} \leq\left\|X u^{a}\right\|_{2}+\|X \Delta u\|_{2},
$$

and hence that $\|X\|_{\text {op }} \leq \max _{u^{a} \in \mathcal{A}}\left\|X u^{a}\right\|_{2}+\frac{1}{2}\|X\|_{\text {op }}$. Re-arranging yields the useful inequality

$$
\|X\|_{\text {op }} \leq 2 \max _{u^{a} \in \mathcal{A}}\left\|X u^{a}\right\|_{2} .
$$

Using inequality (G.1), we have

$$
\begin{aligned}
\mathbb{P}\left[\frac{1}{n}\left\|X^{T} X\right\|_{\text {op }}>t\right] & \leq \mathbb{P}\left[\max _{u^{a} \in \mathcal{A}} \frac{1}{n} \sum_{i=1}^{n}\left(\left\langle u^{a}, X_{i}\right\rangle\right)^{2}>\frac{t}{2}\right] \\
& \leq 4^{m} \max _{u^{a} \in \mathcal{A}} \mathbb{P}\left[\frac{1}{n} \sum_{i=1}^{n}\left(\left\langle u^{a}, X_{i}\right\rangle\right)^{2}>\frac{t}{2}\right] .
\end{aligned}
$$

where the last inequality follows from the union bound, and the fact $[4,5]$ that there exists a $1 / 2$-covering of $S^{m-1}$ with at most $4^{m}$ elements.

In order to complete the proof, we need to obtain a sharp upper bound on the quantity $\mathbb{P}\left[\frac{1}{n} \sum_{i=1}^{n}\left(\left\langle u, X_{i}\right\rangle\right)^{2}>\frac{t}{2}\right]$, valid for any fixed $u \in S^{m-1}$. Define the random vector $Y \in \mathbb{R}^{n}$ with elements $Y_{i}=\left\langle u, X_{i}\right\rangle$. Note that $Y$ is zero mean, and its covariance matrix $R$ has elements $R_{i j}=\mathbb{E}\left[Y_{i} Y_{j}\right]=u^{T} \Sigma\left(\Theta^{*}\right)^{|j-i|} u$. In order to bound the spectral norm of $R$, we note that since it is symmetric, we have $\|R\|_{\text {op }} \leq \max _{i=1, \ldots, m} \sum_{j=1}^{m}\left|R_{i j}\right|$, and moreover

$$
\left|R_{i j}\right|=\left|u^{T} \Sigma\left(\Theta^{*}\right)^{|j-i|} u\right| \leq\left(\left\|\Theta^{*}\right\|_{\text {op }}\right)^{|j-i|} \Sigma \leq \gamma^{|j-i|}\|\Sigma\|_{\text {op }} .
$$

Combining the pieces, we obtain

$$
\|R\|_{\text {op }} \leq \max _{i} \sum_{j=1}^{m}|\gamma|^{|i-j|}\|\Sigma\|_{\text {op }} \leq 2\|\Sigma\|_{\text {op }} \sum_{j=0}^{\infty}|\gamma|^{j} \leq \frac{2\|\Sigma\|_{\text {op }}}{1-\gamma} .
$$

Moreover, we have $\operatorname{trace}(R) / n=u^{T} \Sigma u \leq\|\Sigma\|_{\text {op }}$. Applying Lemma I.2 with $t=5 \sqrt{\frac{m}{n}}$, we conclude that

$$
\left.\mathbb{P}\left[\frac{1}{n}\|Y\|_{2}^{2}>\|\Sigma\|_{\text {op }}+5 \sqrt{\frac{m}{n}}\|R\|_{\text {op }}\right] \leq 2 \exp (-5 m)+2 \exp -n / 2\right) . .
$$


Combined with the bound (G.2), we obtain

$$
\left\|\frac{1}{n} X^{T} X\right\|_{\text {op }} \leq\|\Sigma\|_{\text {op }}\left\{2+\frac{20}{(1-\gamma)} \sqrt{\frac{m}{n}}\right\} \leq \frac{24\|\Sigma\|_{\text {op }}}{(1-\gamma)},
$$

with probability at least $1-c_{1} \exp \left(-c_{2} m\right)$, which establishes the upper bound (35)(a).

Turning to the lower bound (35)(b), we let $\mathcal{B}=\left\{v^{1}, \ldots, v^{B}\right\}$ be an $\epsilon$ cover of $S^{m-1}$ for some $\epsilon \in(0,1)$ to be chosen. Thus, for any $v \in \mathbb{R}^{m}$, there exists some $v^{b}$ such that $v=v^{b}+\Delta v$, and $\|\Delta v\|_{2} \leq \epsilon$. Define the function $\Psi: \mathbb{R}^{m} \times \mathbb{R}^{m} \rightarrow \mathbb{R}$ via $\Psi(u, v)=u^{T}\left(\frac{1}{n} X^{T} X\right) v$, and note that $\Psi(u, v)=\Psi(v, u)$. With this notation, we have

$$
\begin{aligned}
v^{T}\left(\frac{1}{n} X^{T} X\right) v=\Psi(v, v) & =\Psi\left(v^{k}, v^{k}\right)+2 \Psi(\Delta v, v)+\Psi(\Delta v, \Delta v) \\
& \geq \Psi\left(v^{k}, v^{k}\right)+2 \Psi(\Delta v, v),
\end{aligned}
$$

since $\Psi(\Delta v, \Delta v) \geq 0$. Since $|\Psi(\Delta v, v)| \leq \epsilon\left\|\left(\frac{1}{n} X^{T} X\right)\right\|_{\text {op }}$, we obtain the lower bound

$\sigma_{\min }\left(\left(\frac{1}{n} X^{T} X\right)\right)=\inf _{v \in S^{m-1}} v^{T}\left(\frac{1}{n} X^{T} X\right) v \geq \min _{v^{b} \in \mathcal{B}} \Psi\left(v^{b}, v^{b}\right)-2 \epsilon\left\|\frac{1}{n} X^{T} X\right\|_{\mathrm{op}}$.

By the previously established upper bound(35)(a), have $\left\|\frac{1}{n} X^{T} X\right\|_{\text {op }} \leq \frac{24\|\Sigma\|_{\text {op }}}{(1-\gamma)}$ with high probability. Hence, choosing $\epsilon=\frac{(1-\gamma) \sigma_{\min }(\Sigma)}{200\|\Sigma\|_{\text {op }}}$ ensures that $2 \epsilon\left\|\frac{1}{n} X^{T} X\right\|_{\text {op }} \leq \sigma_{\min }(\Sigma) / 4$.

Consequently, it suffices to lower bound the minimum over the covering set. We first establish a concentration result for the function $\Psi(v, v)$ that holds for any fixed $v \in S^{m-1}$. Note that we can write

$$
\Psi(v, v)=\frac{1}{n} \sum_{i=1}^{n}\left(\left\langle v, X_{i}\right\rangle\right)^{2},
$$

As before, if we define the random vector $Y \in \mathbb{R}^{n}$ with elements $Y_{i}=\left\langle v, X_{i}\right\rangle$, then $Y \sim N(0, R)$ with $\|R\|_{\text {op }} \leq \frac{2\|\Sigma\|_{\text {op }}}{1-\gamma}$. Moreover, we have trace $(R) / n=$ $v^{T} \Sigma v \geq \sigma_{\min }(\Sigma)$. Consequently, applying Lemma I.2 yields

$\mathbb{P}\left[\frac{1}{n}\|Y\|_{2}^{2}<\sigma_{\min }(\Sigma)-\frac{8 t\|\Sigma\|_{\text {op }}}{1-\gamma}\right] \leq 2 \exp \left(-n(t-2 / \sqrt{n})^{2} / 2\right)+2 \exp \left(-\frac{n}{2}\right)$,

Note that this bound holds for any fixed $v \in S^{m-1}$. Setting $t^{*}=\frac{(1-\gamma) \sigma_{\min }(\Sigma)}{16\|\Sigma\|_{\text {op }}}$ and applying the union bound yields that

$\mathbb{P}\left[\min _{v^{b} \in \mathcal{B}} \Psi\left(v^{b}, v^{b}\right)<\sigma_{\min }(\Sigma) / 2\right] \leq\left(\frac{4}{\epsilon}\right)^{m}\left\{2 \exp \left(-n\left(t^{*}-2 / \sqrt{n}\right)^{2} / 2\right)+2 \exp \left(-\frac{n}{2}\right)\right\}$, 
which vanishes as long as $n>\frac{4 \log (4 / \epsilon)}{\left(t^{*}\right)^{2}} m$.

G.2. Proof of Lemma 5. Let $S^{m-1}=\left\{u \in \mathbb{R}^{m} \mid\|u\|_{2}=1\right\}$ denote the Euclidean sphere in $m$-dimensions, and for positive scalars $a$ and $b$, define the random variable

$$
\Psi(a, b):=\sup _{u \in a S^{m-1}} \sup _{v \in b S^{m-1}}\langle X v, W u\rangle .
$$

Note that our goal is to upper bound $\Psi(1,1)$. Let $\mathcal{A}=\left\{u^{1}, \ldots, u^{A}\right\}$ and $\mathcal{B}=\left\{v^{1}, \ldots, v^{B}\right\}$ denote $1 / 4$ coverings of $S^{m-1}$ and $S^{m-1}$, respectively. Following the same argument as in the proof of Lemma 3, we obtain the upper bound

$$
\Psi(1,1) \leq 4 \max _{u^{a} \in \mathcal{A}, v^{b} \in \mathcal{B}}\left\langle X v^{b}, W u^{a}\right\rangle
$$

We now apply the union bound to control the discrete maximum. It is known (e.g., $[4,5])$ that there exists a $1 / 4$ covering of $S^{m-1}$ with at most $8^{m}$ elements. Consequently, we have

$$
\mathbb{P}[|\psi(1,1)| \geq 4 \delta n] \leq 8^{2 m} \max _{u^{a}, v^{b}} \mathbb{P}\left[\frac{\left|\left\langle X v^{b}, W u^{a}\right\rangle\right|}{n} \geq \delta\right] .
$$

It remains to obtain a tail bound on the quantity $\mathbb{P}\left[\frac{|\langle X v, W u\rangle|}{n} \geq \delta\right]$, for any fixed pair $(u, v) \in \mathcal{A} \times \mathcal{B}$.

For each $i=1, \ldots, n$, let $X_{i}$ and $W_{i}$ denote the $i^{\text {th }}$ row of $X$ and $W$. Following some simple algebra, we have the decomposition $\frac{\langle X v, W u\rangle}{n}=T_{1}-$ $T_{2}-T_{3}$, where

$$
\begin{aligned}
& T_{1}=\frac{1}{2 n} \sum_{i=1}^{n}\left(\left\langle u, W_{i}\right\rangle+\left\langle v, X_{i}\right\rangle\right)^{2}-\frac{1}{2}\left(u^{T} C u+v^{T} \Sigma v\right) \\
& T_{2}=\frac{1}{2 n} \sum_{i=1}^{n}\left(\left\langle u, W_{i}\right\rangle\right)^{2}-\frac{1}{2} u^{T} C u \\
& T_{3}=\frac{1}{2 n} \sum_{i=1}^{n}\left(\left\langle v, X_{i}\right\rangle\right)^{2}-\frac{1}{2} v^{T} \Sigma v
\end{aligned}
$$

We may now bound each $T_{j}$ for $j=1,2,3$ in turn; in doing so, we make repeated use of Lemma I.2, which provides concentration bounds for a random variable of the form $\|Y\|_{2}^{2}$, where $Y \sim N(0, Q)$ for some matrix $Q \succeq 0$. 
Bound on $T_{3}:$. We can write the term $T_{3}$ as a deviation of $\|Y\|_{2}^{2} / n$ from its mean, where in this case the covariance matrix $Q$ is no longer the identity. In concrete terms, let us define a random vector $Y \in \mathbb{R}^{n}$ with elements $Y_{i}:=$ $\left\langle v, X_{i}\right\rangle$. As seen in the proof of Lemma 4 from Appendix G.1, the vector $Y$ is zero-mean Gaussian with covariance matrix $R$ such that $\|R\|_{\text {op }} \leq \frac{2\|\Sigma\|_{\text {op }}}{1-\gamma}$ (see equation (G.3)). Since we have trace $(R) / n=v^{T} R v$, applying Lemma I.2 yields that

$$
\mathbb{P}\left[\left|T_{3}\right| \geq \frac{8\|\Sigma\|_{\mathrm{op}}}{1-\gamma} t\right] \leq 2 \exp \left(-\frac{n(t-2 / \sqrt{n})^{2}}{2}\right)+2 \exp (-n / 2) .
$$

Bound on $T_{2}:$ We control the term $T_{2}$ in a similar way. Define the random vector $Y^{\prime} \in \mathbb{R}^{n}$ with elements $Y_{i}^{\prime}:=\left\langle u, W_{i}\right\rangle$. Then $Y$ is a sample from the distribution $N\left(0,\left(u^{T} C u\right) I_{n \times n}\right)$, so that $\frac{2}{u^{T} C u} T_{2}$ is the difference between a rescaled $\chi^{2}$ variable and its mean. Applying Lemma I.2 with $Q=\left(u^{T} C u\right) I$, we obtain

$$
\mathbb{P}\left[\left|T_{2}\right|>4\left(u^{T} C u\right) t\right] \leq 2 \exp \left(-\frac{n(t-2 / \sqrt{n})^{2}}{2}\right)+2 \exp (-n / 2) .
$$

Bound on $T_{1} \therefore$ To control this quantity, let us define a zero-mean Gaussian random vector $Z \in \mathbb{R}^{n}$ with elements $Z_{i}=\left\langle v, X_{i}\right\rangle+\left\langle u, W_{i}\right\rangle$. This random vector has covariance matrix $S$ with elements

$$
S_{i j}=\mathbb{E}\left[Z_{i} Z_{j}\right]=\left(u^{T} C u\right) \delta_{i j}+\left(1-\delta_{i j}\right)\left(u^{T} C u\right) v^{T}\left(\Theta^{*}\right)^{|i-j|-1} u+v^{T}\left(\Theta^{*}\right)^{|i-j|} \Sigma v,
$$

where $\delta_{i j}$ is the Kronecker delta for the event $\{i=j\}$. As before, by symmetry of $S$, we have $\|S\|_{\text {op }} \leq \max _{i=1, \ldots, n} \sum_{j=1}^{n}\left|S_{i j}\right|$, and hence

$$
\begin{aligned}
\|S\|_{\text {op }} \leq\left(u^{T} C u\right)+\| & \Sigma \|_{\text {op }}+\sum_{j=1}^{i-1}\left|\left(u^{T} C u\right) v^{T}\left(\Theta^{*}\right)^{|i-j|-1} u+v^{T}\left(\Theta^{*}\right)^{|i-j|} \Sigma v\right| \\
& +\sum_{j=i+1}^{n}\left|\left(u^{T} C u\right) v^{T}\left(\Theta^{*}\right)^{|i-j|-1} u+v^{T}\left(\Theta^{*}\right)^{|i-j|} \Sigma v\right| .
\end{aligned}
$$

Since $\left\|\Theta^{*}\right\|_{\text {op }} \leq \gamma<1$, and $\left(u^{T} C u\right) \leq\|C\|_{\text {op }} \leq\|\Sigma\|_{\text {op }}$, we have

$$
\begin{aligned}
\|S\|_{\text {op }} & \leq\|C\|_{\text {op }}+\|\Sigma\|_{\text {op }}+2 \sum_{j=1}^{\infty}\|C\|_{\text {op }} \gamma^{j-1}+2 \sum_{j=1}^{\infty}\|\Sigma\|_{\text {op }} \gamma^{j} \\
& \leq 4\|\Sigma\|_{\text {op }}\left(1+\frac{1}{1-\gamma}\right)
\end{aligned}
$$

imsart-aos ver. 2010/08/03 file: RR_AOS_Sahand_trim.tex date: November 16, 2010 
Moreover, we have $\frac{\operatorname{trace}(S)}{n}=\left(u^{T} C u\right)+v^{T} \Sigma v \leq 2\|\Sigma\|_{\text {op }}$, so that by applying Lemma I.2, we conclude that

$$
\mathbb{P}\left[\left|T_{1}\right|>\left(\frac{24\|\Sigma\|_{\mathrm{op}}}{1-\gamma}\right) t\right] \leq 2 \exp \left(-\frac{n(t-2 / \sqrt{n})^{2}}{2}\right)+2 \exp (-n / 2)
$$

which completes the analysis of this term.

Combining the bounds (G.7), (G.8) and (G.9), we conclude that for all $t>0$,

$$
\mathbb{P}\left[\frac{|\langle X v, W u\rangle|}{n} \geq \frac{40\left(\|\Sigma\|_{\text {op }} t\right)}{1-\gamma}\right] \leq 6 \exp \left(-\frac{n(t-2 / \sqrt{n})^{2}}{2}\right)+6 \exp (-n / 2) .
$$

Setting $t=10 \sqrt{m / n}$ and combining with the bound (G.6), we conclude that $\mathbb{P}\left[|\psi(1,1)| \geq \frac{1600\|\Sigma\|_{\text {op }}}{1-\gamma} \sqrt{\frac{m}{n}}\right] \leq 8^{2 m}\{6 \exp (-16 m)+6 \exp (-n / 2)\} \leq 12 \exp (-m)$ as long as $n>((4 \log 8)+1) m$.

\section{APPENDIX H: PROOF OF PROPOSITION 1}

We begin by stating and proving a useful lemma. Recall the definition (22) of $\rho(\Sigma)$.

Lemma H.1. Let $X \in \mathbb{R}^{m_{1} \times m_{2}}$ be a random sample from the $\Sigma$-ensemble. Then we have

$$
\mathbb{E}\left[\|X\|_{\text {op }}\right] \leq 12 \rho(\Sigma)\left[\sqrt{m_{1}}+\sqrt{m_{2}}\right]
$$

and moreover

$$
\mathbb{P}\left[\|X\|_{\text {op }} \geq \mathbb{E}\left[\|X\|_{\text {op }}\right]+t\right] \leq \exp \left(-\frac{t^{2}}{2 \rho^{2}(\Sigma)}\right) .
$$

Proof. We begin by making note of the variational representation

$$
\|X\|_{\text {op }}=\sup _{(u, v) \in S^{m_{1}-1} \times S^{m_{2}-1}} u^{T} X v .
$$

Since each variable $u^{T} X v$ is zero-mean Gaussian, we thus recognize $\|X\|_{\text {op }}$ as the supremum of a Gaussian process. The bound (H.2) thus follows from Theorem 7.1 in Ledoux [6]. 
We now use a simple covering argument establish the upper bound (H.1). Let $\left\{v^{1}, \ldots, v^{M_{2}}\right\}$ be a $1 / 4$ covering of the sphere $S^{m_{2}-1}$. For an arbitrary $v \in S^{m_{2}-1}$, there exists some $v^{j}$ in the cover such that $\left\|v-v^{j}\right\|_{2} \leq 1 / 4$, whence

$$
\|X v\|_{2} \leq\left\|X v^{j}\right\|_{2}+\left\|X\left(v-v_{j}\right)\right\|_{2} .
$$

Taking suprema over both sides, we obtain that $\|X\|_{\text {op }} \leq \max _{j=1, \ldots, M_{2}}\left\|X v^{j}\right\|_{2}+$ $\frac{1}{4}\|X\|_{\text {op }}$. A similar argument using a $1 / 4$-covering $\left\{u^{1}, \ldots, u^{M_{1}}\right\}$ of $S^{m_{1}-1}$ yields that

$$
\left\|X v^{j}\right\|_{2} \leq \max _{i=1, \ldots, M_{1}}\left\langle u^{i}, X v^{j}\right\rangle+\frac{1}{4}\|X\|_{\mathrm{op}} .
$$

Combining the pieces, we conclude that

$$
\|X\|_{\mathrm{op}} \leq 2 \max _{\substack{i=1, \ldots, M_{1} \\ j=1, \ldots, M_{2}}}\left\langle u^{i}, X v^{j}\right\rangle .
$$

By construction, each variable $\left\langle u^{i}, X v^{j}\right\rangle$ is zero-mean Gaussian with variance at most $\rho(\Sigma)$, so that by standard bounds on Gaussian maxima, we obtain

$$
\mathbb{E}\left[\|X\|_{\text {op }}\right] \leq 4 \rho(\Sigma) \sqrt{\log \left(M_{1} M_{2}\right)} \leq 4 \rho(\Sigma)\left[\sqrt{\log M_{1}}+\sqrt{\log M_{2}}\right] .
$$

There exist 1/4-coverings of $S^{m_{1}-1}$ and $S^{m_{2}-1}$ with $\log M_{1} \leq m_{1} \log 8$ and $\log M_{2} \leq m_{2} \log 8$, from which the bound (H.1) follows.

We now return to the proof of Proposition 1. To simplify the proof, let us define an operator $T_{\Sigma}: \mathbb{R}^{m_{1} \times m_{2}} \rightarrow \mathbb{R}^{m_{1} \times m_{2}}$ such that $\operatorname{vec}\left(T_{\Sigma}(\Theta)\right)=$ $\sqrt{\Sigma} \operatorname{vec}(\Theta)$. Let $\mathfrak{X}^{\prime}: \mathbb{R}^{m_{1} \times m_{2}} \rightarrow \mathbb{R}^{N}$ be a random Gaussian operator formed with $X_{i}^{\prime}$ sampled with i.i.d. $N(0,1)$ entries. By construction, we then have $\mathfrak{X}(\Theta)=\mathfrak{X}^{\prime}\left(T_{\Sigma}(\Theta)\right)$ for all $\Theta \in \mathbb{R}^{m_{1} \times m_{2}}$. Now by the variational characterization of the $\ell_{2}$-norm, we have

$$
\left\|\mathfrak{X}^{\prime}\left(T_{\Sigma}(\Theta)\right)\right\|_{2}=\sup _{u \in S^{N-1}}\left\langle u, \mathfrak{X}^{\prime}\left(T_{\Sigma}(\Theta)\right)\right\rangle .
$$

Since the original claim (25) is invariant to rescaling, it suffices to prove it for matrices such that $\left\|T_{\Sigma}(\Theta)\right\|_{F}=1$. Letting $t \geq 1$ be a given radius, we seek lower bounds on the quantity

$$
\begin{aligned}
\left.Z^{*}(t):=\inf _{\Theta \in \mathcal{R}(t)} \sup _{u \in S^{N-1}}\left\langle u, \mathfrak{X}^{\prime}\left(T_{\Sigma}(\Theta)\right)\right)\right\rangle, \\
\quad \text { where } \mathcal{R}(t)=\left\{\Theta \in \mathbb{R}^{m_{1} \times m_{2}} \mid\left\|T_{\Sigma}(\Theta)\right\|_{F}=1,\|\Theta\|_{1} \leq t\right\} .
\end{aligned}
$$


In particular, our goal is to prove that for any $t \geq 1$, the lower bound

$$
\frac{Z^{*}(t)}{\sqrt{N}} \geq \frac{1}{4}-12 \rho(\Sigma)\left[\frac{m_{1}+m_{2}}{N}\right]^{1 / 2} t
$$

holds with probability at least $1-c_{1} \exp \left(-c_{2} N\right)$. By a standard peeling argument (see Raskutti et al. [7] for details), this lower bound implies the claim (25).

We establish the lower bound (H.3) using Gaussian comparison inequalities [4] and concentration of measure (see Lemma I.1). For each pair $(u, \Theta) \in$ $S^{N-1} \times \mathcal{R}(t)$, consider the random variable $Z_{u, \Theta}=\left\langle u, \mathfrak{X}^{\prime}\left(T_{\Sigma}(\Theta)\right)\right\rangle$, and note that it is Gaussian with zero mean. For any two pairs $(u, \Theta)$ and $\left(u^{\prime}, \Theta^{\prime}\right)$, some calculation yields

$$
\mathbb{E}\left[\left(Z_{u, \Theta}-Z_{u^{\prime}, \Theta^{\prime}}\right)^{2}\right]=\left\|u \otimes T_{\Sigma}(\Theta)-u^{\prime} \otimes T_{\Sigma}\left(\Theta^{\prime}\right)\right\|_{F}^{2} .
$$

We now define a second Gaussian process $\left\{Y_{u, \Theta} \mid(u, \Theta) \in S^{N-1} \times \mathcal{R}(t)\right\}$ via

$$
Y_{u, \Theta}:=\langle g, u\rangle+\left\langle\left\langle G, T_{\Sigma}(\Theta)\right\rangle\right\rangle,
$$

where $g \in \mathbb{R}^{N}$ and $G \in \mathbb{R}^{m_{1} \times m_{2}}$ are independent with i.i.d. $N(0,1)$ entries. By construction, $Y_{u, \Theta}$ is zero-mean, and moreover, for any two pairs $(u, \Theta)$ and $\left(u^{\prime}, \Theta^{\prime}\right)$, we have

$$
\mathbb{E}\left[\left(Y_{u, \Theta}-Y_{u^{\prime}, \Theta^{\prime}}\right)^{2}\right]=\left\|u-u^{\prime}\right\|_{2}^{2}+\left\|T_{\Sigma}(\Theta)-T_{\Sigma}\left(\Theta^{\prime}\right)\right\|_{F}^{2} .
$$

For all pairs $(u, \Theta),\left(u^{\prime}, \Theta^{\prime}\right) \in S^{N-1} \times \mathcal{R}(t)$, we have $\|u\|_{2}=\left\|u^{\prime}\right\|_{2}=1$, and moreover $\left\|T_{\Sigma}(\Theta)\right\|_{F}=\left\|T_{\Sigma}\left(\Theta^{\prime}\right)\right\|_{F}=1$. Using this fact, some algebra yields that

(H.6) $\quad\left\|u \otimes T_{\Sigma}(\Theta)-u^{\prime} \otimes T_{\Sigma}\left(\Theta^{\prime}\right)\right\|_{F}^{2} \leq\left\|u-u^{\prime}\right\|_{2}^{2}+\left\|T_{\Sigma}(\Theta)-T_{\Sigma}\left(\Theta^{\prime}\right)\right\|_{F}^{2}$.

Moreover, equality holds whenever $\Theta=\Theta^{\prime}$. The conditions of the GordonSlepian inequality [4] are satisfied, so that we are guaranteed that

$$
\mathbb{E}\left[\inf _{\Theta \in \mathcal{R}(t)}\left\|\mathfrak{X}^{\prime}\left(T_{\Sigma}(\Theta)\right)\right\|_{2}\right]=\mathbb{E}\left[\inf _{\Theta \in \mathcal{R}(t)} \sup _{u \in S^{N-1}} Z_{u, \Theta}\right] \geq \mathbb{E}\left[\inf _{\Theta \in \mathcal{R}(t)} \sup _{u \in S^{N-1}} Y_{u, \Theta}\right]
$$

We compute

$$
\begin{aligned}
\mathbb{E}\left[\inf _{\Theta \in \mathcal{R}(t)} \sup _{u \in S^{N-1}} Y_{u, \Theta}\right] & =\mathbb{E}\left[\sup _{u \in S^{N-1}}\langle g, u\rangle\right]+\mathbb{E}\left[\inf _{\Theta \in \mathcal{R}(t)}\left\langle\left\langle G, T_{\Sigma}(\Theta)\right\rangle\right]\right] \\
& =\mathbb{E}\left[\|g\|_{2}\right]-\mathbb{E}\left[\sup _{\Theta \in \mathcal{R}(t)}\left\langle\left\langle G, T_{\Sigma}(\Theta)\right\rangle\right\rangle\right] \\
& \geq \frac{1}{2} \sqrt{N}-t \mathbb{E}\left[\left\|T_{\Sigma}(G)\right\|_{\mathrm{op}}\right],
\end{aligned}
$$

imsart-aos ver. 2010/08/03 file: RR_AOS_Sahand_trim.tex date: November 16, 2010 
where we have used the fact that $T_{\Sigma}$ is self-adjoint, and Hölder's inequality (involving the operator and nuclear norms). Since $T_{\Sigma}(G)$ is a random matrix from the $\Sigma$-ensemble, Lemma H.1 yields the upper bound $\mathbb{E}\left[\left\|T_{\Sigma}(G)\right\|_{\text {op }}\right] \leq$ $12 \rho(\Sigma)\left(\sqrt{m_{1}}+\sqrt{m_{2}}\right)$. Putting together the pieces, we conclude that

$$
\mathbb{E}\left[\inf _{\Theta \in \mathcal{R}(t)} \frac{\left\|\mathfrak{X}^{\prime}\left(T_{\Sigma}(\Theta)\right)\right\|_{2}}{\sqrt{N}}\right] \geq \frac{1}{2}-12 \rho(\Sigma)\left(\frac{\sqrt{m_{1}}+\sqrt{m_{2}}}{\sqrt{N}}\right) t .
$$

Finally, we need to establish sharp concentration around the mean. Since $\left\|T_{\Sigma}(\Theta)\right\|_{F}=1$ for all $\Theta \in \mathcal{R}(t)$, the function $f(\mathfrak{X}):=\inf _{\Theta \in \mathcal{R}(t)}\left\|\mathfrak{X}^{\prime}\left(T_{\Sigma}(\Theta)\right)\right\|_{2} / \sqrt{N}$ is Lipschitz with constant $1 / \sqrt{N}$, so that Lemma I.1 implies that

$$
\mathbb{P}\left[\inf _{\Theta \in \mathcal{R}(t)} \frac{\|\mathfrak{X}(\Theta)\|_{2}}{\sqrt{N}} \leq \frac{1}{2}-12 \rho(\Sigma)\left(\frac{\sqrt{m_{1}}+\sqrt{m_{2}}}{\sqrt{N}}\right) t-\delta\right] \leq 2 \exp \left(-N \delta^{2} / 2\right)
$$

for all $\delta>0$. Setting $\delta=1 / 4$ yields the claim.

\section{APPENDIX I: SOME USEFUL CONCENTRATION RESULTS}

The following lemma is classical $[4,8]$, and yields sharp concentration of a Lipschitz function of Gaussian random variables around its mean.

Lemma I.1. Let $X \in \mathbb{R}^{n}$ have i.i.d. $N(0,1)$ entries, and let and $f: \mathbb{R}^{n} \rightarrow$ $\mathbb{R}$ be Lipschitz with constant $L$ (i.e., $|f(x)-f(y)| \leq L\|x-y\|_{2} \forall x, y \in \mathbb{R}^{n}$ ). Then for all $t>0$, we have

$$
\mathbb{P}[|f(X)-E f(X)|>t] \leq 2 \exp \left(-\frac{t^{2}}{2 L^{2}}\right) .
$$

By exploiting this lemma, we can prove the following result, which yields concentration of the squared $\ell_{2}$-norm of an arbitrary Gaussian vector:

Lemma I.2. Given a Gaussian random vector $Y \sim N(0, Q)$, for all $t>$ $2 / \sqrt{n}$, we have

$\mathbb{P}\left[\frac{1}{n}\left|\|Y\|_{2}^{2}-\operatorname{trace} Q\right|>4 t\|Q\|_{\text {op }}\right] \leq 2 \exp \left(-\frac{n\left(t-\frac{2}{\sqrt{n}}\right)^{2}}{2}\right)+2 \exp (-n / 2)$.

Proof. Let $\sqrt{Q}$ be the symmetric matrix square root, and consider the function $f(x)=\|\sqrt{Q} x\|_{2} / \sqrt{n}$. Since it is Lipschitz with constant $\|\sqrt{Q}\|_{\mathrm{op}} / \sqrt{n}$, Lemma I.1 implies that

$\mathbb{P}\left[\left|\|\sqrt{Q} X\|_{2}-E\|\sqrt{Q} X\|_{2}\right|>\sqrt{n} \delta\right] \leq 2 \exp \left(-\frac{n \delta^{2}}{2\|Q\| \text { op }}\right) \quad$ for all $\delta>0$.

imsart-aos ver. 2010/08/03 file: RR_AOS_Sahand_trim.tex date: November 16, 2010 
By integrating this tail bound, we find that the variable $Z=\|\sqrt{Q} X\|_{2} / \sqrt{n}$ satisfies the bound $\operatorname{var}(Z) \leq 4\|Q\|_{\text {op }} / n$, and hence conclude that

$$
\left|\sqrt{\mathbb{E}\left[Z^{2}\right]}-\right| \mathbb{E}[Z] \||=| \sqrt{\operatorname{trace}(Q) / n}-\mathbb{E}\left[\|\sqrt{Q} X\|_{2} / \sqrt{n}\right] \mid \leq \frac{2 \sqrt{\|Q\|_{\mathrm{op}}}}{\sqrt{n}} .
$$

Combining this bound with the tail bound (I.2), we conclude that

$\mathbb{P}\left[\frac{1}{\sqrt{n}}\left|\|\sqrt{Q} X\|_{2}-\sqrt{\operatorname{trace}(Q)}\right|>\delta+2 \sqrt{\frac{\|Q\|_{\mathrm{op}}}{n}}\right] \leq 2 \exp \left(-\frac{n \delta^{2}}{2\|Q\|_{\mathrm{op}}}\right) \quad$ for all $\delta>0$.

Setting $\delta=(t-2 / \sqrt{n}) \sqrt{\|Q\|_{\text {op }}}$ in the bound (I.4) yields that

$$
\mathbb{P}\left[\frac{1}{\sqrt{n}}\left|\|\sqrt{Q} X\|_{2}-\sqrt{\operatorname{trace}(Q)}\right|>t \sqrt{\|Q\|_{\text {op }}}\right] \leq 2 \exp \left(-\frac{n(t-2 / \sqrt{n})^{2}}{2}\right) .
$$

Similarly, setting $\delta=\sqrt{\|Q\|_{\text {op }}}$ in the tail bound (I.4) yields that with probability greater than $1-2 \exp (-n / 2)$, we have

$$
\left|\frac{\|Y\|_{2}}{\sqrt{n}}+\sqrt{\frac{\operatorname{trace}(Q)}{n}}\right| \leq \sqrt{\frac{\operatorname{trace}(Q)}{n}}+3 \sqrt{\|Q\|_{\text {op }}} \leq 4 \sqrt{\|Q\|_{\text {op }}} .
$$

Using these two bounds, we obtain

$$
\left|\frac{\|Y\|_{2}^{2}}{n}-\frac{\operatorname{trace}(Q)}{n}\right|=\left|\frac{\|Y\|_{2}}{\sqrt{n}}-\sqrt{\frac{\operatorname{trace}(Q)}{n}}\right|\left|\frac{\|Y\|_{2}}{\sqrt{n}}+\sqrt{\frac{\operatorname{trace}(Q)}{n}}\right| \leq 4 t\|Q\|_{\text {op }}
$$

with the claimed probability.

\section{REFERENCES}

[1] S. Negahban and M. J. Wainwright. Estimation of (near) low-rank matrices with noise and high-dimensional scaling. Technical Report http://arxiv.org/abs/0912.5100, UC Berkeley, Department of Statistics, December 2009.

[2] B. Recht, M. Fazel, and P. A. Parrilo. Guaranteed minimum-rank solutions of linear matrix equations via nuclear norm minimization. SIAM Review, 2007. to appear.

[3] S. Boyd and L. Vandenberghe. Convex optimization. Cambridge University Press, Cambridge, UK, 2004.

[4] M. Ledoux and M. Talagrand. Probability in Banach Spaces: Isoperimetry and Processes. Springer-Verlag, New York, NY, 1991. 
[5] J. Matousek. Lectures on discrete geometry. Springer-Verlag, New York, 2002.

[6] M. Ledoux. The Concentration of Measure Phenomenon. Mathematical Surveys and Monographs. American Mathematical Society, Providence, RI, 2001.

[7] G. Raskutti, M. J. Wainwright, and B. Yu. Minimax rates of estimation for highdimensional linear regression over $\ell_{q}$-balls. Technical Report arXiv:0910.2042, UC Berkeley, Department of Statistics, 2009. Presented in part at Allerton Conference, Sep. 2009.

[8] P. Massart. Concentration Inequalties and Model Selection. Ecole d'Eté de Probabilités, Saint-Flour. Springer, New York, 2003.

Department of Electrical EngineERing

University of California, Berkeley

Berkeley, CA 94720

USA

E-MAIL: sahand_n@eecs.berkeley.edu
Department of Statistics

University of CAlifornia, Berkeley

Berkeley, California 94720

USA

E-MAIL: wainwrig@eecs.berkeley.edu 